Authors are encouraged to submit new papers to INFORMS journals by means of a style file template, which includes the journal title. However, use of a template does not certify that the paper has been accepted for publication in the named journal. INFORMS journal templates are for the exclusive purpose of submitting to an INFORMS journal and should not be used to distribute the papers in print or online or to submit the papers to another publication.

\title{
Expressiveness and Robustness of First-Price Position Auctions
}

\author{
Paul Dütting \\ Department of Mathematics, London School of Economics, Houghton Street, London WC2A 2AE, UK \\ p.d.duetting@lse.ac.uk \\ Felix Fischer \\ School of Computing Science, University of Glasgow, Sir Alwyn Williams Building, Glasgow G12 8QQ, UK \\ fischerf@dcs.gla.ac.uk \\ David C. Parkes \\ Harvard John A. Paulson School of Engineering and Applied Sciences, Harvard University, \\ 33 Oxford Street, Cambridge, MA 02138, USA \\ parkes@eecs.harvard.edu
}

\begin{abstract}
Ideally the properties of an economic mechanism should hold in a robust way across multiple equilibria and under varying assumptions regarding the information available to participants. Focusing on the design of robust position auctions we seek mechanisms that possess an efficient equilibrium, and guarantee high revenue in every efficient equilibrium, under both complete and incomplete information. A generalized first-price auction that is expressive in the sense of allowing multi-dimensional bids turns out to be the only standard design able to achieve this goal, even when valuations are one-dimensional. The equilibria under complete information are obtained via Bernheim and Whinston's profit-target strategies, those under incomplete information via an appropriate generalization thereof. Particularly interesting from a technical perspective is the incomplete-information case, where the standard technique for establishing equilibrium existence due to Myerson is generalized to a setting in which the bid space has higher dimension than the valuation space.

Key words: simplicity-expressiveness tradeoffs, generalized first-price auction, profit-target strategies MSC2000 subject classification: Primary: 91B26; secondary: 91A10

OR/MS subject classification: Primary: games/group decisions, bidding/auctions; secondary: games/group decisions, noncooperative
\end{abstract}

1. Introduction. We consider a standard position auction setting in which $k$ positions are to be assigned to $n$ agents in a one-to-one fashion and agents agree on the relative values of the positions. Such a setting can be described by two vectors $\boldsymbol{v}=\left(v_{1}, \ldots, v_{n}\right)$ and $\boldsymbol{\beta}=\left(\beta_{1}, \ldots, \beta_{k}\right)$, where $v_{i}$ is private to agent $i$ and $\boldsymbol{\beta}$ is publicly known. The valuation of agent $i$ for position $j$ is then given by $\beta_{j} v_{i}$, and we will assume for convenience that $\beta_{1} \geq \beta_{2} \geq \cdots \geq \beta_{k}$. This is a one-dimensional setting, as the private information of each agent consists of a single number. A prime example of a position auction can be found in the context of sponsored search, where agents correspond to advertisers and positions to slots in which advertisements are displayed. Each slot comes with a click-through rate and each advertiser with a value per click.

In position auction settings, the same auction format is often applied across very different problem instances. Internet search engines for example use the same format to auction off both valu- 
able high-frequency keywords and a large number of keywords corresponding to less frequent user queries. For high-frequency keywords it is reasonable to consider a complete-information model, where agents know one another's value per click. For less frequent keywords, on the other hand, an incomplete-information model, where an agent only has probabilistic beliefs about the values of others, seems more appropriate. An auction design used for both high- and low-frequency keywords should therefore possess appropriate strategic equilibria under both complete and incomplete information.

In addition to the question of equilibrium existence, auction designs are subject to a tradeoff among various performance criteria. A common goal is the maximization of social welfare, i.e., the sum of agents' valuations for the positions they are assigned. This goal corresponds to an efficient use of the available resources, which is desirable for the long-term health of a marketplace. From the auctioneer's point of view, the revenue achieved by the auction should also be robust in the sense that it does not vary too much among different efficient equilibria, if indeed there is more than one such equilibrium. We thus arrive at the following question:

Does there exist a position auction that always admits an efficient equilibrium, and achieves high revenue in every efficient equilibrium, under both complete and incomplete information?

The Vickrey-Clarke-Groves (VCG) auction possesses an efficient equilibrium in which agents truthfully reveal their valuations, but it also admits additional efficient equilibria with low revenue when agents are sufficiently well informed about one another's valuations [34, 16]. The truthful equilibrium of the VCG mechanism will nevertheless play a central role in our analysis in that we adopt the corresponding revenue, henceforth termed the truthful VCG revenue, as a benchmark. Under complete information this benchmark is well justified and has been used in previous work [e.g., 32]. It is also a sensible choice under incomplete information, where it is the maximum revenue achievable by an efficient mechanism $[35,30] .{ }^{1}$

Before we proceed any further, it is worth noting that revenue equivalence, which states that equilibria resulting in the same allocation must also yield the same revenue, is not enough to resolve the question under either complete or incomplete information: under complete information revenue equivalence does not generally hold, under incomplete information it does not guarantee the existence of an efficient equilibrium.

1.1. Candidate auctions. We address the question by considering variants of the three standard designs for position auctions: the Vickrey-Clarke-Groves (VCG) auction, the generalized second-price (GSP) auction, and the generalized first-price (GFP) auction. ${ }^{2}$ For each of these designs we distinguish between a simplified version with one-dimensional bids and an expressive version with multi-dimensional bids. In the simplified version each agent $i$ specifies a single bid $b_{i}$, and this bid is multiplied by $\beta_{1} \geq \beta_{2} \geq \cdots \geq \beta_{k}$ to obtain bids for the different positions. In the expressive version each agent $i$ submits a separate bid $b_{i j}$ for each position $j$.

We further distinguish between two allocation rules: the first assigns positions to agents so as to maximize social welfare with regard to the reported valuations; the second considers each position in turn, from first to last, and greedily assigns it to an agent with maximum bid for the position among those not previously assigned a position. For simplified bids the greedy allocation rule also maximizes reported social welfare and the two allocation rules are identical. This is not generally

\footnotetext{
${ }^{1}$ An alternative benchmark for settings with incomplete information, the optimal revenue achievable by any auction, sacrifices efficiency [35]. This seems inappropriate given our focus on efficient equilibria.

2 The GSP auction is used by Google and Microsoft. The GFP auction was used by Overture, the first company to provide a successful sponsored search service. Facebook uses the VCG auction to place ads, although not in a context where positions can always clearly be ranked by value.
} 
Dütting, Fischer, and Parkes: Expressiveness and Robustness of First-Price Position Auctions

TABLE 1. Negative results for standard auction designs and focus of this article

\begin{tabular}{|c|c|c|c|c|}
\hline \multirow[t]{2}{*}{ bid space } & \multirow[t]{2}{*}{ allocation rule } & \multicolumn{3}{|l|}{ payment rule } \\
\hline & & VCG-based & GSP-based & GFP-based \\
\hline simplified & $\begin{array}{l}\text { efficient } \equiv \\
\text { greedy }\end{array}$ & $\begin{array}{l}\text { efficient low-revenue } \\
\text { Nash equilibrium [16] }\end{array}$ & $\begin{array}{l}\text { efficient low-revenue } \\
\text { Nash equilibrium [16], } \\
\text { no Bayes-Nash equi- } \\
\text { librium [25] }\end{array}$ & $\begin{array}{l}\text { no Nash } \\
\text { equilibrium [21] }\end{array}$ \\
\hline \multirow{2}{*}{ expressive } & efficient & $\begin{array}{l}\text { efficient low-revenue } \\
\text { Nash equilibrium [34] }\end{array}$ & $\begin{array}{l}\text { efficient low-revenue } \\
\text { Nash equilibrium [34] }\end{array}$ & \\
\hline & greedy & $\begin{array}{l}\text { efficient low-revenue } \\
\text { Nash equilibrium [34] }\end{array}$ & $\begin{array}{l}\text { efficient low-revenue } \\
\text { Nash equilibrium [34] }\end{array}$ & this article \\
\hline
\end{tabular}

the case for expressive bids. Our positive results exploit the simpler structure of outcomes under the greedy allocation rule, ${ }^{3}$ while all negative results hold for both allocation rules.

For a given allocation rule, payments are finally defined as follows: the VCG auction charges each agent the difference in social welfare of the other agents when the agent is absent and when it is present, both with regard to reported valuations; the GSP auction charges each agent the next-highest bid on the position it is assigned coming from an agent that is not assigned a higher position; the GFP auction charges each agent its bid on the position it is assigned.

1.2. Results. It turns out that most candidate auctions are disqualified by prior results, as shown in Table 1. All versions of the VCG and GSP auctions - with simplified or expressive bids, efficient or greedy allocation rule - have an efficient complete-information equilibrium with arbitrarily small revenue compared to the truthful VCG revenue [34, 16]. This result is quite robust and continues to hold for example when the auctioneer uses incorrect multipliers $\boldsymbol{\alpha} \neq \boldsymbol{\beta}$ in place of $\boldsymbol{\beta}$ [16]. An additional disadvantage of the simplified GSP auction is that it may not have any (Bayes-Nash) equilibrium when information is incomplete [25]. The simplified GFP auction, on the other hand, has a unique (Bayes-Nash) equilibrium under incomplete information [11], but may not have an equilibrium under complete information [21].

The only remaining candidate is the expressive GFP auction, and we show that the version with a greedy allocation rule indeed possesses the desired properties. Under complete information it has an efficient equilibrium, and all of its equilibria are efficient and yield at least the truthful VCG revenue. Under incomplete information it has an efficient equilibrium, and every efficient equilibrium yields the truthful VCG revenue.

Efficient equilibria in the two cases can be obtained via profit-target strategies, a notion proposed by Bernheim and Whinston [6] for settings with complete information, ${ }^{4}$ and via an appropriate generalization thereof to settings with incomplete information. In following a profit-target strategy an agent uses its knowledge of the valuations of others to determine a profit it can achieve and then bids in a way that guarantees this profit no matter which allocation is reached. We generalize the idea to settings with incomplete information by replacing profit by expected profit and the uniform target by a per-allocation target, as befits new uncertainty regarding the allocation eventually selected. We also observe that such conditioning on the allocation is in fact necessary to obtain an equilibrium, even in a single-item auction.

${ }^{3}$ An allocation maximizing reported social welfare corresponds to a maximum-weight matching, which means in particular that the decision to allocate position $j$ to agent $i$ depends on the bids of all agents on all positions. For the greedy allocation rule this decision is independent of bids on positions $\ell>j$.

${ }^{4}$ Bernheim and Whinston in fact refer to the strategies in question as "truthful," Ausubel and Milgrom [3] as "semisincere." Milgrom [33] argues that the term profit-target strategy is more appropriate, because agents submit bids that guarantee them a certain utility or profit in case they are allocated. 
Interestingly, it is the same target that leads to an efficient equilibrium under both complete and incomplete information, namely the utility each agent would obtain in the truthful equilibrium of the VCG auction. In both cases it can be reached only via multi-dimensional bids, which explains why expressiveness beyond the valuation space is necessary. That not only the same mechanism can be used in both settings, but that the same approach to bidding leads to an equilibrium, is the best outcome one might hope for from a practical perspective. It means that both the auctioneer and the bidders can follow the same approach irrespective of the actual amount of information available to the bidders.

1.3. Techniques. Our analysis of the complete-information case is technically similar to the classic analysis of Bernheim and Whinston [6] that links equilibria of first-price auctions to the core, and to more recent approaches that also make this connection [14, 29]. The common feature is the use of profit-target strategies, and we show specifically that using the truthful VCG utility of each agent as its target utility yields an efficient equilibrium. By construction, revenue in this equilibrium equals the truthful VCG revenue. We then exclude the possibility of inefficient equilibria by showing how inefficiencies in the allocation create opportunities for beneficial unilateral deviation. To establish the revenue guarantee for efficient equilibria we consider certain unilateral deviations from the proposed efficient equilibrium and use the fact that these deviations cannot be beneficial to derive lower bounds on payments in equilibrium.

In the incomplete-information setting, the combination of one-dimensional valuations and multidimensional bids poses two technical challenges. Firstly, Myerson's [35] equilibrium characterization only provides a necessary but not a sufficient condition on possible equilibrium bids: it tells us that payments in every efficient equilibrium must equal those in the truthful equilibrium of the VCG auction. However, since bids are multi-dimensional, there are many different bids that satisfy the condition and thus many candidate equilibrium bids. Secondly, the standard technique to verify that a particular candidate is an equilibrium involves integrating the derivative of an agent's utility, as a function of both valuation and bid, along a path between two bids. This technique breaks down in our setting, where the bid space has higher dimension than the valuation space and the utility function may not be defined everywhere on the path.

We address the first difficulty through an appropriate generalization of the profit-target idea from complete to incomplete information and obtain a strategy where an agent's bid on a given position equals its expected truthful VCG payment under the condition of being allocated that position. We then establish that these bids constitute an equilibrium, and thus overcome the second difficulty, by showing that for a particular agent and any position $j$ it is optimal to bid according to the conjectured equilibrium on position $j$, given that the other agents bid according to the conjectured equilibrium and the agent bids according to the conjectured equilibrium on positions $j+1$ to $k$. We believe that similar techniques could be used to show equilibrium existence in more general settings, including settings with multi-dimensional valuations.

1.4. Related work. The design of expressive auctions for specific applications is an important topic of contemporary mechanism design [e.g., 2, 23, 13, 20, 15, 18, 24]. The intuition that expressiveness is generally desirable is supported by results of Benisch et al. [5], who showed that the maximum social welfare a mechanism can achieve strictly increases with a measure of expressiveness based on a concept from computational learning theory.

The classic analysis of position auctions is due to Varian [37] and Edelman et al. [21]. Working under the assumption that agents have complete information, the authors showed that the GSP auction - although not truthful - has certain desirable equilibria, and that revenue in these equilibria is at least that in the truthful equilibrium of the VCG auction. Under incomplete information, the GSP auction may not possess an efficient equilibrium [25]. The GFP auction, on the other 
hand, always has a unique, efficient (Bayes-Nash) equilibrium under incomplete information, which yields the truthful VCG revenue [11], but may not have a (Nash) equilibrium under complete information [21]. Neither the simplified GSP auction nor the simplified GFP auction should thus be expected to perform well under both complete and incomplete information. However, in a situation where one of them does perform well, it may do so more widely than the VCG auction once the latter's truthfulness is compromised [17].

Under both complete and incomplete information, the social welfare in any equilibrium of the GSP auction is within a constant factor of the maximum social welfare $[10,36]$. The revenue of the GSP auction can be arbitrarily small compared to the truthful VCG revenue under complete information, whereas under incomplete information it is always within a constant factor of the optimal revenue of Myerson [35] when reserve prices are chosen appropriately [32, 9].

To the best of our knowledge, the study of position auctions that admit efficient equilibria and yield high revenue in every efficient equilibrium under both complete and incomplete information, and the use of additional expressiveness to achieve this goal, is novel to the present work. Our analysis differs from earlier results in its use of an expressive bidding language to overcome the negative result for complete-information settings.

The role of expressiveness in position-auction environments was first highlighted by Milgrom [34] and Dütting et al. [16], who considered VCG and GSP auctions under complete information and argued that a restriction of the bidding space to a subspace of the valuation space can rule out zero-revenue equilibria without introducing new and potentially undesirable ones. Also in a complete-information setting and for VCG and GSP auctions, Blumrosen et al. [8] and Abrams et al. [1] bounded the reduction in equilibrium quality resulting from a restriction of the bidding space to a subspace of the valuation space.

Independently from our work, and again focusing on complete-information environments, Hoy et al. [29] argued in favor of expressive designs for first-price auctions. For an auction enabling the explicit revelation of target utilities the authors showed that an efficient equilibrium resulting in the truthful VCG revenue is guaranteed to exist, and that every equilibrium satisfying an additional envy-freeness property is efficient and yields the truthful VCG revenue. Our results for completeinformation environments do not require envy-freeness for the latter to be the case. In addition we also provide an analysis for settings with incomplete information, in what we believe is the first application of the notion of profit-target strategies in such settings.

2. Preliminaries. We study a setting with a set $\{1, \ldots, k\}$ of positions ordered by quality and a set $\{1, \ldots, n\}$ of agents with unit demand and one-dimensional valuations for the positions. More formally, let $\mathbb{R}_{>}^{k}=\left\{\boldsymbol{x} \in \mathbb{R}^{k}: x_{j} \geq 0, x_{j} \geq x_{j^{\prime}}\right.$ if $\left.j<j^{\prime}\right\}$ be the set of $k$-dimensional vectors whose entries are non-negative and non-increasing. Denote $\mathbb{R}_{+}=\{x \in \mathbb{R}: x \geq 0\}$. For $\boldsymbol{\beta} \in \mathbb{R}_{\geq}^{k}$, let $\mathbb{R}_{\boldsymbol{\beta}}^{k}=\left\{\boldsymbol{x} \in \mathbb{R}^{k}: \boldsymbol{x}=\boldsymbol{\beta} v, v \in \mathbb{R}_{+}\right\}$be the one-dimensional subspace of $\mathbb{R}_{+}^{k}$ spanned by $\boldsymbol{\beta}$. The valuation of agent $i$ can then be represented by a vector $\boldsymbol{\beta} v_{i} \in \mathbb{R}_{\boldsymbol{\beta}}^{k}$ in this subspace, such that $\beta_{j} v_{i} \geq 0$ is the agent's value for position $j$. We assume without loss of generality that $\beta_{k}>0$ and $n>k .^{5}$

Our goal is to produce a one-to-one assignment of positions to agents that is efficient in the sense that it maximizes social welfare, the sum of agents' valuations for the positions they are assigned. To this end, the $V C G$ auction solicits a one-dimensional bid $b_{i} \in \mathbb{R}_{+}$from each agent $i \in N$. This bid is then interpreted as a $k$-dimensional bid by multiplication with $\boldsymbol{\beta}$, such that the bid of agent $i$ for position $j$ is $\beta_{j} v_{i}$, and the allocation is chosen to maximize the reported social welfare, i.e., the sum of the bids for positions assigned. The payment of an agent is the amount

\footnotetext{
${ }^{5}$ A setting where $\beta_{k}=0$ is equivalent to a setting with $k-1$ positions. A setting with $k>n$ positions is equivalent to a setting with $n$ positions, as the greedy allocation rule never assigns the other positions. A setting where $k=n$ finally is equivalent to a setting with $k^{\prime}<n$ positions, where $k^{\prime}=\arg \max \left\{i: \beta_{i}-\beta_{k}>0\right\}$, as in the former each agent $i$ is guaranteed a value of at least $\beta_{k} v_{i}$.
} 
by which its presence reduces the reported social welfare of the other agents. Assuming agents are ordered such that $b_{1} \geq b_{2} \geq \cdots \geq b_{n}$, agent $i$ is assigned position $i$ and pays $\sum_{s=i}^{k}\left(\beta_{s}-\beta_{s+1}\right) b_{s+1}$. The expressive GFP auction solicits a vector $\boldsymbol{b}_{i} \in \mathbb{R}_{+}^{k}$ of bids from each agent $i \in N$, where $b_{i j}$ is interpreted as agent $i$ 's bid on position $j$. The allocation is determined in a greedy manner by considering positions 1 to $k$ in turn, and assigning the current position to an agent with maximum bid on that position among the agents not assigned an earlier position. The payment of an agent $i$ assigned position $j$ is its bid $b_{i j}$ on that position.

When reasoning about strategic behavior by the agents we assume quasi-linear utilities, such that the utility $u_{i}\left(\boldsymbol{b}, v_{i}\right)$ of agent $i$ with value $v_{i}$ in a given auction and for a given bid profile $\boldsymbol{b}=\left(\boldsymbol{b}_{1}, \ldots, \boldsymbol{b}_{n}\right)$ is equal to its valuation for the position it is assigned minus its payment for that position, and use two models for the information available to the agents regarding one another's valuations. Both models assume $\boldsymbol{\beta}$ to be common knowledge among the agents. In the completeinformation model the same is true also for the values $v_{i}$, and a bid profile $\left(\boldsymbol{b}_{1}, \ldots, \boldsymbol{b}_{n}\right)$ is a Nash equilibrium of an auction if no agent has an incentive to change its bid assuming that the other agents don't change their bids, i.e., if for every $i \in N$ and every $\boldsymbol{b}_{i}^{\prime} \in \mathbb{R}_{+}^{k}$,

$$
u_{i}\left(\left(\boldsymbol{b}_{1}, \ldots, \boldsymbol{b}_{i}, \ldots, \boldsymbol{b}_{n}\right), v_{i}\right) \geq u_{i}\left(\left(\boldsymbol{b}_{1}, \ldots, \boldsymbol{b}_{i}^{\prime}, \ldots, \boldsymbol{b}_{n}\right), v_{i}\right) .
$$

The incomplete-information model assumes instead that the values $v_{i}$ are drawn independently from a continuous distribution $F$ with density $f$ supported on $[0, \bar{v}]$ for some finite $\bar{v} \in \mathbb{R}_{+}$, and that $F$ is common knowledge among the agents. ${ }^{6}$ The strategy of a particular agent can then be represented in terms of a bidding function $\boldsymbol{b}: \mathbb{R}_{+} \rightarrow \mathbb{R}_{+}^{k}$ that maps the agent's value $v$ to a vector $\boldsymbol{b}(v)=\left(b_{1}(v), \ldots, b_{k}(v)\right)$ of bids on positions 1 to $k$.

It is not difficult to see that to obtain an efficient allocation with probability one, the bidding functions of all agents must be identical except on a set of measure zero. Indeed, consider a situation where two different bidding functions $\boldsymbol{b}$ and $\boldsymbol{b}^{\prime}$ are being used and let $j$ be the first position where the two functions differ. Then there must exist $v, v^{\prime}$ with $v<v^{\prime}$ and $b_{j}(v)>b_{j}^{\prime}\left(v^{\prime}\right)$, which for appropriately chosen valuations leads to a contradiction to efficiency. Bidding function $\boldsymbol{b}$ then is a Bayes-Nash equilibrium if no agent has an incentive to unilaterally change its bid assuming that all other agents have valuations drawn from $F$ and bid according to $\boldsymbol{b}$, i.e., if for every $i \in N$, every $v_{i} \in[0, \bar{v}]$, and every bidding function $\boldsymbol{b}^{\prime}$,

$$
\begin{aligned}
\mathbb{E}_{v_{j} \sim F, j \neq i}\left[u_{i}\left(\left(\boldsymbol{b}\left(v_{1}\right), \ldots, \boldsymbol{b}\left(v_{i-1}\right), \boldsymbol{b}\left(v_{i}\right), \boldsymbol{b}\left(v_{i+1}\right), \ldots, \boldsymbol{b}\left(v_{n}\right)\right), v_{i}\right)\right] \geq \\
\mathbb{E}_{v_{j} \sim F, j \neq i}\left[u_{i}\left(\left(\boldsymbol{b}\left(v_{1}\right), \ldots, \boldsymbol{b}\left(v_{i-1}\right), \boldsymbol{b}^{\prime}\left(v_{i}\right), \boldsymbol{b}\left(v_{i+1}\right), \ldots, \boldsymbol{b}\left(v_{n}\right)\right), v_{i}\right)\right] .
\end{aligned}
$$

3. Complete information. We begin our analysis of the expressive GFP auction for settings with complete information and show that it always possesses a Nash equilibrium, that all its Nash equilibria are efficient, and that payments in any Nash equilibrium are at least the truthful VCG payments. The first result is established via profit-target strategies where each agent targets its utility in the truthful equilibrium of the VCG auction. The bid of agent $i$ on position $j$ then equals its value $\beta_{j} v_{i}$ minus its truthful VCG utility, or zero if this difference is negative. ${ }^{7}$ For the second result we argue that any inefficiency in the allocation creates an opportunity for beneficial unilateral deviation. The proof of the third result uses the non-existence of beneficial unilateral deviations in a Nash equilibrium to derive lower bounds on payments. ${ }^{8}$

\footnotetext{
${ }^{6}$ The assumption of boundedness is made for notational convenience. Our results also hold for example for distributions with finite expectation.

${ }^{7}$ It is worth noting that a one-dimensional bid will not usually be enough to express the target utility for each position, even if the target utility is identical across positions.

${ }^{8}$ An alternative proof could use the connections between the VCG outcome and Walrasian equilibria [26] and between Walrasian equilibria and equilibria of expressive first-price auctions [27]. Our proof from first principles has the advantage that it makes the role of profit-target strategies explicit.
} 
THEOREM 1. In a position auction with complete information, the expressive GFP auction with greedy allocation rule has the following properties:

1. it possesses an efficient Nash equilibrium in which the payment of every agent is the same as in the truthful equilibrium of the VCG auction;

2. all of its Nash equilibria are efficient;

3. in all of its Nash equilibria the payment of every agent is at least its payment in the truthful equilibrium of the VCG auction.

Proof. We prove each of the claims in turn.

For the first claim, consider without loss of generality the case where agents are ordered by nonincreasing value, such that $v_{1} \geq v_{2} \geq \cdots \geq v_{n}$, and the efficient allocation where agent $i$ is assigned position $i$, for $1 \leq i \leq k$. Denote by $u_{i}$ the truthful VCG utility of agent $i$, for $1 \leq i \leq n$, and by $p_{i}$ the truthful VCG payment for position $i$, for $1 \leq i \leq k$. Then $u_{i}=\beta_{i} v_{i}-p_{i}$ if $1 \leq i \leq k$ and $u_{i}=0$ if $i>k$. We further claim that the bid profile $\boldsymbol{b}$ with

$$
b_{i j}=\max \left(\beta_{j} v_{i}-u_{i}, 0\right)
$$

for $i=1, \ldots, n$ and $j=1, \ldots, k$ is an equilibrium of the GFP auction that is efficient and yields the truthful VCG payments.

Under this bid profile an efficient allocation assigns position $i$ to agent $i$ at price $p_{i}$. With the greedy allocation rule, this outcome can be obtained by letting agent $i$ point to position $i$ and breaking ties in favor of the agent that points to a given position if only one agent points to the position and in an arbitrary but fixed manner if more than one agent points to it. To see that $\boldsymbol{b}$ is indeed an equilibrium, first observe that agent $i$ cannot decrease its bid for position $i$ without being assigned a position other than $i$. Now assume for contradiction that agent $i$ has a beneficial deviation to a position $j \neq i$, such that

$$
\beta_{i} v_{i}-p_{i}<\beta_{j} v_{i}-p_{j}-\epsilon,
$$

for every $\epsilon>0$. Here we use that agent $i$ can bid $p_{j}+\epsilon$ on positions $j$ and above to be assigned one of these positions, and that it values each of them at least as highly as position $j$. The left-hand side of this inequality equals the utility of agent $i$ in the truthful equilibrium of the VCG auction, whereas the right-hand side equals the utility agent $i$ would obtain if it was instead assigned position $j$ at price $p_{j}+\epsilon$. The inequality contradicts the fact that the truthful VCG equilibrium is envy-free [31].

For the second claim, consider a Nash equilibrium $\boldsymbol{b}=\left(\boldsymbol{b}_{1}, \ldots, \boldsymbol{b}_{n}\right)$ and assume for contradiction that it leads to an inefficient assignment. Then there exist agents $i$ and $j$ with $v_{i}>v_{j}$ such that agent $i$ is assigned position $s$ and agent $j$ is assigned position $t<s$ such that $\beta_{t}>\beta_{s}$.

First assume that agent $i$ bids $b_{j t}+\epsilon$ on positions $t$ and above, which means that it is assigned one of these positions. Since $\boldsymbol{b}$ is an equilibrium this deviation is not beneficial, i.e., for every $\epsilon>0$,

$$
\beta_{s} v_{i}-b_{i s} \geq \beta_{t} v_{i}-b_{j t}-\epsilon
$$

Now consider the situation where agent $j$ bids according to bid vector $\boldsymbol{b}_{j}^{\prime}$ with

$$
b_{j, \ell}^{\prime}= \begin{cases}b_{i s}+\epsilon & \text { if } 1 \leq \ell \leq s \\ 0 & \text { otherwise }\end{cases}
$$

for some $\epsilon>0$. We claim that with these bids agent $j$ will either be assigned a position above $s$, or will compete for position $s$ with bids that are at most $b_{i s}$ and will therefore be assigned position $s$. For the latter observe that because assignments are made greedily, agents other than $j$ who are assigned a position above $s$ when agent $j$ bids according to $\boldsymbol{b}_{j}$ can only be assigned a higher 
position when agent $j$ bids according to $\boldsymbol{b}_{j}^{\prime}$. This suffices because agents other than $j$ who were assigned position $s$ or below bid at most $b_{i s}$ on position $s$.

Since $\boldsymbol{b}$ is an equilibrium agent $j$ does not benefit from bidding according to $\boldsymbol{b}_{j}^{\prime}$, and thus for every $\epsilon>0$,

$$
\beta_{t} v_{j}-b_{j t} \geq \beta_{s} v_{j}-b_{i s}-\epsilon .
$$

By adding (1) and (2) and rearranging,

$$
\beta_{s} v_{i}+\beta_{t} v_{j} \geq \beta_{s} v_{j}+\beta_{t} v_{i}-2 \epsilon,
$$

and thus

$$
v_{j} \geq v_{i}-\frac{2 \epsilon}{\beta_{t}-\beta_{s}},
$$

where we have used that $\beta_{t}-\beta_{s}>0$. Since the inequality holds for every $\epsilon>0$, we obtain a contradiction to the assumption that $v_{i}>v_{j}$.

For the third claim, consider a Nash equilibrium $\boldsymbol{b}=\left(\boldsymbol{b}_{1}, \ldots, \boldsymbol{b}_{n}\right)$ and assume without loss of generality that it leads to an assignment where agent $i$ is assigned position $i$ for $1 \leq i \leq k$. Further assume that the assignment is efficient, i.e., that $v_{1} \geq v_{2} \geq \cdots \geq v_{k}$. For $1 \leq i \leq k$, agent $i+1$ does not benefit from bidding $b_{i, i}+\epsilon=p_{i}+\epsilon$ on position $i$ and above, so for every $\epsilon>0$,

$$
\beta_{i+1} v_{i+1}-p_{i+1} \geq \beta_{i} v_{i+1}-p_{i}-\epsilon
$$

and thus

$$
\begin{aligned}
& p_{k} \geq \beta_{k} v_{k+1}-\epsilon, \quad \text { and } \\
& p_{i} \geq\left(\beta_{i}-\beta_{i+1}\right) v_{i+1}+p_{i+1}-\epsilon \quad \text { for } 1 \leq i<k .
\end{aligned}
$$

This proves the claim.

4. Incomplete information. We now turn to settings with incomplete information and recall that an efficient Bayes-Nash equilibrium must be symmetric in the sense that all agents use the same bidding function. Moreover, by Myerson's characterization [35], the expected payment of an agent must be the same in any efficient equilibrium of any mechanism, and identical in particular to that in the efficient equilibrium of the VCG auction in which each agent bids its true valuation. While this gives us an indication of equilibrium payments and thus of potential equilibrium bidding functions, it only needs to hold in expectation and therefore does not determine the bidding function uniquely.

Let us first see why a direct translation of the approach we used under complete information fails under incomplete information. To this end consider a single-item auction and two agents with valuations distributed uniformly on the unit interval. The expected truthful VCG utility of an agent with valuation $v$ then is $u=v^{2} / 2$. However, the bid $b$ such that the utility upon allocation coincides with $u$, i.e., $b=\max \{v-u, 0\}=v-v^{2} / 2$, does not meet the requirements of Myerson's characterization and thus cannot be an equilibrium. Indeed $b$ is too high, or equivalently the corresponding target utility $u$ too low. Intuitively the agent should target a higher utility, as the fact that it is assigned the item reveals additional information about its valuation relative to that of the other agent.

This example suggests that in a position auction, the target utility upon allocation of a particular position must differ among positions, and the most obvious way to achieve such a conditioning is to set the bid $b_{j}^{*}(v)$ of an agent with value $v$ for position $j$ to be equal to the expected payment an agent with this value would face if in the truthful equilibrium of the VCG auction it was allocated position $j$. The expectation here is taken over the values of the other agents and conditioned on $v$ being the $j$-highest among all values. The bidding function $\boldsymbol{b}^{*}: \mathbb{R}_{+} \rightarrow \mathbb{R}_{+}^{k}$ such that $\boldsymbol{b}^{*}(v)=$ $\left(b_{i}^{*}(v), \ldots, b_{k}^{*}(v)\right)$ turns out to be an equilibrium. 
THEOREM 2. In a position auction with incomplete information and values drawn independently from a continuous distribution with bounded support, the expressive GFP auction with greedy allocation rule has an efficient Bayes-Nash equilibrium in which the expected payment of every agent is equal to its payment in the truthful equilibrium of the VCG auction.

We will define $\boldsymbol{b}^{*}$ formally in Section 4.1 and call $b_{j}^{*}(v)$ the straightforward bid of an agent with value $v$ on position $j$. To establish the theorem we use three lemmas, which we prove respectively in Sections 4.2, 4.3, and 4.4. The first lemma states that for each $j, b_{j}^{*}$ is continuous and strictly increasing.

Lemma 1. For $j \in\{1 \ldots k\}, b_{j}^{*}$ is continuous on $[0, \bar{v}]$. For $j \in\{1 \ldots k\}$ and $v \in(0, \bar{v})$,

$$
\frac{d}{d v} b_{j}^{*}(v)>0
$$

For $v \in \mathbb{R}_{+}$and $\boldsymbol{x} \in \mathbb{R}_{+}^{k}$, denote by $u^{*}\left(\left(x_{1}, \ldots, x_{k}\right), v\right)$ the expected utility of an agent with value $v$ who bids $b_{j}^{*}\left(x_{j}\right)$ on position $j$ while all other agents bid straightforwardly. The second and third lemma concern two properties of $u^{*}$ as a function of the agent's value $v$ and its bid $b_{j}^{*}\left(x_{j}\right)$ on position $j$, assuming straightforward bids on positions $j+1$ to $k$ : for any $v$, it is stationary at the straightforward bid $b_{j}^{*}(v)$; and it changes more rapidly in $x_{j}$ as $v$ increases, given that straightforward bidding on positions $j+1$ to $k$ is optimal irrespective of the bids on positions 1 to $k$.

Lemma 2. For $j \in\{1 \ldots k\}, v \in(0, \bar{v}]$, and $x_{1}, \ldots, x_{j-1} \in \mathbb{R}_{+}$,

$$
\left.\frac{d}{d x_{j}} u^{*}\left(\left(x_{1}, \ldots, x_{j}, v, \ldots, v\right), v\right)\right|_{x_{j}=v}=0
$$

Lemma 3. Let $j \in\{1, \ldots, k\}$ such that for all $v \in[0, \bar{v}]$ and all $\boldsymbol{x} \in \mathbb{R}_{+}^{k}, u^{*}\left(\left(x_{1}, \ldots, x_{j}, v, \ldots, v\right), v\right) \geq$ $u^{*}(\boldsymbol{x}, v)$. Let $v \in[0, \bar{v}]$ and $x_{1}, \ldots, x_{j-1} \in \mathbb{R}_{+}$. Then

$$
\frac{d}{d v} \frac{d}{d x_{j}} u^{*}\left(\left(x_{1}, \ldots, x_{j-1}, x_{j}, v, \ldots, v\right), v\right) \geq 0 .
$$

We are now ready to prove the theorem.

Proof of Theorem 2. That bidding according to $\boldsymbol{b}^{*}$ leads to an efficient assignment follows from Lemma 1 and the fact that positions are assigned greedily. The claim concerning the payments then holds by definition of $\boldsymbol{b}^{*}$ and because each agent pays its bid on the position it is assigned.

For the equilibrium property consider a situation where $n-1$ agents bid according to $\boldsymbol{b}^{*}$. Note that for the remaining agent it is enough to consider bids $b_{j}^{*}(v)$ where $v$ is in the support of $F$, since any other bid is dominated by a bid of this type. Let $j \geq 0$ be the minimum value such that for all $v$ and all $\boldsymbol{x}, u^{*}\left(\left(x_{1}, \ldots, x_{j}, v, \ldots, v\right), v\right) \geq u^{*}(\boldsymbol{x}, v)$, and note that $\boldsymbol{b}^{*}$ is an equilibrium when $j=0$. Assume for contradiction that $j>0$ and consider $v \in \mathbb{R}_{+}$and $\boldsymbol{x} \in \mathbb{R}_{+}^{k}$ such that $x_{j} \neq v$ and

$$
u^{*}\left(\left(x_{1}, \ldots, x_{j-1}, x_{j}, v, \ldots, v\right), v\right)>u^{*}\left(\left(x_{1}, \ldots, x_{j-1}, v, v, \ldots, v\right), v\right) .
$$

From continuity of $b_{j}^{*}$, which holds by Lemma 1 , it follows that $u^{*}\left(\left(x_{1}, \ldots, x_{j-1}, x_{j}, v, \ldots, v\right), v\right)$ is continuous in both $x_{j}$ and $v$. We can thus assume without loss of generality that $x_{j}>0$ and $v>0$. 
If $x_{j}<v$, then

$$
\begin{aligned}
u^{*}\left(\left(x_{1}, \ldots, x_{j-1}, v, v, \ldots, v\right), v\right) & -u^{*}\left(\left(x_{1}, \ldots, x_{j-1}, x_{j}, v, \ldots, v\right), v\right) \\
& =\int_{y=x_{j}}^{v} \frac{d}{d y} u^{*}\left(\left(x_{1}, \ldots, x_{j-1}, y, v, \ldots, v\right), v\right) d y \\
& \geq\left.\int_{y=x_{j}}^{v}\left(\frac{d}{d z} u^{*}\left(\left(x_{1}, \ldots, x_{j-1}, z, y, \ldots, y\right), y\right)\right)\right|_{z=y} d z=0,
\end{aligned}
$$

which is a contradiction. Here the inequality holds because by Lemma 3 , for $y \leq v$,

$$
\frac{d}{d z} u^{*}\left(\left(x_{1}, \ldots, x_{j-1}, z, v, \ldots, v\right), v\right) \geq \frac{d}{d z} u^{*}\left(\left(x_{1}, \ldots, x_{j-1}, z, y, \ldots, y\right), y\right) .
$$

The second equality holds by Lemma 2 .

The same contradiction can also be obtained if $x_{j}>v$, in which case

$$
\begin{aligned}
u^{*}\left(\left(x_{1}, \ldots, x_{j-1}, v, v, \ldots, v\right), v\right) & -u^{*}\left(\left(x_{1}, \ldots, x_{j-1}, x_{j}, v, \ldots, v\right), v\right) \\
& =\int_{y=x_{j}}^{v} \frac{d}{d y} u^{*}\left(\left(x_{1}, \ldots, x_{j-1}, y, v, \ldots, v\right), v\right) d y \\
& =-\int_{y=v}^{x_{j}} \frac{d}{d y} u^{*}\left(\left(x_{1}, \ldots, x_{j-1}, y, v, \ldots, v\right), v\right) d y \\
& \geq-\left.\int_{y=v}^{x_{j}}\left(\frac{d}{d z} u^{*}\left(\left(x_{1}, \ldots, x_{j-1}, z, y, \ldots, y\right), y\right)\right)\right|_{z=y} d z=0 .
\end{aligned}
$$

Here the inequality holds because by Lemma 3 , for $y \geq v$,

$$
-\frac{d}{d z} u^{*}\left(\left(x_{1}, \ldots, x_{j-1}, z, v, \ldots, v\right), v\right) \geq-\frac{d}{d z} u^{*}\left(\left(x_{1}, \ldots, x_{j-1}, z, y, \ldots, y\right), y\right) .
$$

4.1. Truthful VCG payments and allocation probabilities. For $j \in\{1, \ldots, k\}$ and $v \in$ $[0, \bar{v}]$ we defined the straightforward bid $b_{j}^{*}(v)$ on position $j$ of an agent with value $v$ as its expected payment in the truthful equilibrium of the VCG auction, given that it has value $v$ and is allocated position $j$. This quantity is equal to the sum of the differences $\beta_{s}-\beta_{s+1}$ multiplied by the expected value of the $s+1$-highest among $n$ values drawn independently from distribution $F$, and conditioned on $v$ being the $j$-highest value. Thus $b_{j}^{*}(0)=0$ and for $v \in(0, \bar{v}]$,

$$
b_{j}^{*}(v)=\sum_{s=j}^{k}\left(\beta_{s}-\beta_{s+1}\right) \int_{u=0}^{v} \frac{(n-j) !}{(n-s-1) !(s-j) !}\left(\frac{F(u)}{F(v)}\right)^{n-s-1}\left(1-\frac{F(u)}{F(v)}\right)^{s-j} \frac{f(u)}{F(v)} u d u .
$$

For $\boldsymbol{x} \in \mathbb{R}_{+}^{k}$ let $P_{s, m}(\boldsymbol{x})$ be the probability that an agent is assigned position $s$ against $m$ other agents who bid straightforwardly if it bids $\boldsymbol{b}^{*}(\boldsymbol{x})=\left(b_{1}^{*}\left(x_{1}\right), \ldots, b_{k}^{*}\left(x_{k}\right)\right)$. Since the agent is assigned position $s$ if $m-s+1$ of the $m$ other agents have values no larger than $x_{s}$ and it is not assigned one of the positions $1, . ., s-1$ against the remaining $s-1$ agents, $P_{s, m}(\boldsymbol{x})$ can be written recursively as

$$
\begin{aligned}
& P_{1, m}(\boldsymbol{x})=F\left(x_{1}\right)^{m}, \quad \text { and } \\
& P_{s, m}(\boldsymbol{x})=\left(\begin{array}{c}
m \\
m-s+1
\end{array}\right) F\left(x_{s}\right)^{m-s+1}\left(1-\sum_{t=1}^{s-1} P_{t, s-1}(\boldsymbol{x})\right) .
\end{aligned}
$$

An important observation is that $P_{s, m}(\boldsymbol{x})$ does not depend on $x_{t}$ for $t>s$. 
4.2. Proof of Lemma 1. Note first that, by the binomial theorem,

$$
\left(1-\frac{F(u)}{F(v)}\right)^{s-j}=\sum_{t=0}^{s-j}(-1)^{t}\left(\begin{array}{c}
s-j \\
t
\end{array}\right)\left(\frac{F(u)}{F(v)}\right)^{t} .
$$

Application to (3) and rearranging yields that for $v>0$,

$$
b_{j}^{*}(v)=\sum_{s=j}^{k} \frac{\left(\beta_{s}-\beta_{s+1}\right)(n-j) !}{(n-s-1) !(s-j) !} \sum_{t=0}^{s-j}(-1)^{t}\left(\begin{array}{c}
s-j \\
t
\end{array}\right) \frac{\int_{u=0}^{v} F(u)^{n-s+t-1} f(u) u d u}{F(v)^{n-s+t}} .
$$

Continuity on $[0, \bar{v}]$ now follows because

$$
\lim _{v \rightarrow 0} b_{j}^{*}(v)=\lim _{v \rightarrow 0} \sum_{s=j}^{k} \frac{\left(\beta_{s}-\beta_{s+1}\right)(n-j) !}{(n-s-1) !(s-j) !} \sum_{t=0}^{s-j}(-1)^{t}\left(\begin{array}{c}
s-j \\
t
\end{array}\right) \frac{F(v)^{n-s+t-1} f(v) v}{(n-s+t) F(v)^{n-s+t-1} f(v)}=0,
$$

where the first equality holds by (6) and by l'Hospital's rule.

Now assume that $v>0$ and let $Z_{n-s+t}(v)=\left(\frac{1}{F(v)}\right)^{n-s+t} \int_{u=0}^{v} F(u)^{n-s+t} d u$. Then

$$
\frac{\int_{u=0}^{v}(n-s+t) F(u)^{n-s+t-1} f(u) u d u}{F(v)^{n-s+t}}=\frac{F(v)^{n-s+t} v-\int_{u=0}^{v} F(u)^{n-s+t} d u}{F(v)^{n-s+t}}=v-Z_{n-s+t}(v),
$$

where the first equality follows by applying integration by parts to the numerator, and thus, by (6),

$$
b_{j}^{*}(v)=\sum_{s=j}^{k}\left(\beta_{s}-\beta_{s+1}\right) \sum_{t=0}^{s-j}(-1)^{t}\left(\begin{array}{c}
s-j \\
t
\end{array}\right) \frac{(n-j) !}{(n-s-1) !(s-j) !} \frac{1}{(n-s+t)}\left(v-Z_{n-s+t}(v)\right) .
$$

Moreover

$$
\begin{aligned}
\frac{d}{d v}\left(v-Z_{n-s+t}(v)\right) & =\frac{d}{d v}\left(v-\left(\frac{1}{F(v)}\right)^{n-s+t} \int_{u=0}^{v} F(u)^{n-s+t} d u\right) \\
& =1+(n-s+t) \frac{f(v)}{F(v)} \frac{1}{F(v)^{n-s+t}} \int_{u=0}^{v} F(u)^{n-s+t} d u-1 \\
& =(n-s+t) \frac{f(v)}{F(v)} Z_{n-s+t}(v),
\end{aligned}
$$

where the first and last equality hold by definition of $Z_{n-s+t}(v)$, and for the second equality we have used the product rule. We now claim that

$$
\begin{aligned}
\frac{d}{d v} b_{j}^{*}(v) & =\sum_{s=j}^{k}\left(\beta_{s}-\beta_{s+1}\right) \sum_{t=0}^{s-j}(-1)^{t}\left(\begin{array}{c}
s-j \\
t
\end{array}\right) \frac{(n-j) !}{(n-s-1) !(s-j) !} \frac{f(v)}{F(v)} Z_{n-s+t}(v) \\
& =\sum_{s=j}^{k}\left(\beta_{s}-\beta_{s+1}\right) \int_{u=0}^{v} \frac{(n-j) !}{(n-s-1) !(s-j) !}\left(1-\frac{F(u)}{F(v)}\right)^{s-j}\left(\frac{F(u)}{F(v)}\right)^{n-s} \frac{f(v)}{F(v)} d u>0 .
\end{aligned}
$$

Indeed the first equality holds by (7) and (8), and the second equality by definition of $Z_{n-s+t}(v)$, by (5), and by rearranging. The inequality follows because the integral is strictly positive for all $s \in\{j, \ldots, k\}$, which is the case since $v>0$ and since $f(v)>0$ by continuity of $F$, and because, by assumption, $\beta_{k}-\beta_{k+1}>0$. 
4.3. Proof of Lemma 2. We write $u^{*}(\boldsymbol{x}, v)$ as a sum of contributions $T_{s}(\boldsymbol{x}, v)=$ $P_{s, n-1}(\boldsymbol{x})\left(\beta_{s} v-b_{s}^{*}\left(x_{s}\right)\right)$ of position $s=1, \ldots, k$, group these contributions into those of positions $s<j$, those of positions $j$ and $j+1$, and those of positions $s>j+1$, and argue for each group separately that the derivative in $x_{j}$ vanishes at $x_{j}=v$.

For the contribution $\sum_{s=1}^{j-1} T_{s}(\boldsymbol{x}, v)$ of positions $s<j$ this is easy, as neither the allocation probability $P_{s, n-1}(\boldsymbol{x})$ nor the utility $\beta_{s} v-b_{s}^{*}\left(x_{s}\right)$ subject to allocation depends on $x_{j}$. Hence the derivative in $x_{j}$ is zero everywhere, and in particular at $x_{j}=v$.

To prove the claim for $T_{j}(\boldsymbol{x}, v)+T_{j+1}(\boldsymbol{x}, v)$, we first apply the recursive formulation of the allocation probabilities to compute the derivatives in $x_{j}$ of $T_{j}(\boldsymbol{x}, v)$ and $T_{j+1}(\boldsymbol{x}, v)$. We then observe that the derivative of $T_{j}(\boldsymbol{x}, v)+T_{j+1}(\boldsymbol{x}, v)$ vanishes at $x_{j}=v$ if a certain differential equation involving the bids $b_{j}^{*}(v)$ and $b_{j+1}^{*}(v)$ is satisfied. To establish that the differential equation does indeed hold we use the following combinatorial identity, which is proved in the appendix.

LEMMA 4. For all $m \in \mathbb{N}$ and $\ell \in \mathbb{N}_{+}$,

$$
\sum_{t=0}^{m}(-1)^{t}\left(\begin{array}{c}
m \\
t
\end{array}\right) \frac{(m+\ell) !}{(\ell-1) ! m !(t+\ell)}=1
$$

Lemma 5. Fix a particular agent with value $v>0$ and a position $j$. Assume that all other agents bid straightforwardly and that the agent bids straightforwardly on positions $j+1, \ldots, k$. Then

$$
\left.\frac{d}{d x_{j}}\left(T_{j}(\boldsymbol{x}, v)+T_{j+1}(\boldsymbol{x}, v)\right)\right|_{x_{j}=v}=0 .
$$

Proof. We begin by considering the contributions $T_{j}(\boldsymbol{x}, v)$ and $T_{j+1}(\boldsymbol{x}, v)$ of positions $j$ and $j+1$ separately. For position $j$,

$$
T_{j}(\boldsymbol{x}, v)=P_{j, n-1}(\boldsymbol{x})\left(\beta_{j} v-b_{j}^{*}\left(x_{j}\right)\right)=\left(\begin{array}{c}
n-1 \\
n-j
\end{array}\right) F\left(x_{j}\right)^{n-j}\left(1-\sum_{t=1}^{j-1} P_{t, j-1}(\boldsymbol{x})\right)\left(\beta_{j} v-b_{j}^{*}\left(x_{j}\right)\right),
$$

where the second equality holds by (4), and thus

$$
\begin{aligned}
\frac{d}{d x_{j}} T_{j}(\boldsymbol{x}, v) & =\left(\begin{array}{l}
n-1 \\
n-j
\end{array}\right)\left(1-\sum_{t=1}^{j-1} P_{t, j-1}(\boldsymbol{x})\right)\left((n-j) F\left(x_{j}\right)^{n-j-1} f\left(x_{j}\right)\left(\beta_{j} v-b_{j}^{*}\left(x_{j}\right)\right)\right. \\
\left.-F\left(x_{j}\right)^{n-j} \frac{d}{d x_{j}} b_{j}^{*}\left(x_{j}\right)\right) & \left.-\frac{F\left(x_{j}\right)}{f\left(x_{j}\right)(n-j)} \frac{d}{d x_{j}} b_{j}^{*}\left(x_{j}\right)\right) .
\end{aligned}
$$

For position $j+1$,

$$
\begin{aligned}
T_{j+1}(\boldsymbol{x}, v) & =P_{j+1, n-1}(\boldsymbol{x})\left(\beta_{j+1} v-b_{j+1}^{*}\left(x_{j+1}\right)\right) \\
& =\left(\begin{array}{c}
n-1 \\
n-j-1
\end{array}\right) F(v)^{n-j-1}\left(1-\sum_{t=1}^{j} P_{t, j}(\boldsymbol{x})\right)\left(\beta_{j+1} v-b_{j+1}^{*}(v)\right) \\
& =\left(\begin{array}{c}
n-1 \\
n-j-1
\end{array}\right) F(v)^{n-j-1}\left(1-\sum_{t=1}^{j-1} P_{t, j}(\boldsymbol{x})-\right. \\
& \left.\left(\begin{array}{l}
j \\
1
\end{array}\right) F\left(x_{j}\right)\left(1-\sum_{t=1}^{j-1} P_{t, j-1}(\boldsymbol{x})\right)\right)\left(\beta_{j+1} v-b_{j+1}^{*}(v)\right),
\end{aligned}
$$


where the second equality holds by (4) and the third equality can be obtained by pulling $P_{j, j}(x)$ out of the sum and applying (4) again. Thus,

$$
\begin{aligned}
\frac{d}{d x_{j}} T_{j+1}(\boldsymbol{x}, v) & =-\left(\begin{array}{c}
n-1 \\
n-j-1
\end{array}\right) F(v)^{n-j-1}\left(\begin{array}{l}
j \\
1
\end{array}\right) f\left(x_{j}\right)\left(1-\sum_{t=1}^{j-1} P_{t, j-1}(\boldsymbol{x})\right)\left(\beta_{j+1} v-b_{j+1}^{*}(v)\right) \\
& =-\left(\begin{array}{c}
n-1 \\
n-j
\end{array}\right)\left(1-\sum_{t=1}^{j-1} P_{t, j-1}(\boldsymbol{x})\right)(n-j) F(v)^{n-j-1} f\left(x_{j}\right)\left(\beta_{j+1} v-b_{j+1}^{*}(v)\right) .
\end{aligned}
$$

From (10) and (11) we see that the claim holds if

$$
\left(\left(\beta_{j} v-b_{j}^{*}(v)\right)-\left.\frac{F(v)}{f(v)(n-j)} \frac{d}{d x_{j}} b_{j}^{*}\left(x_{j}\right)\right|_{x_{j}=v}\right)-\left(\beta_{j+1} v-b_{j+1}^{*}(v)\right)=0
$$

i.e., if

$$
\left.\frac{d}{d x_{j}} b_{j}^{*}\left(x_{j}\right)\right|_{x_{j}=v}=(n-j) \frac{f(v)}{F(v)}\left(\left(\beta_{j} v-b_{j}^{*}(v)\right)-\left(\beta_{j+1} v-b_{j+1}^{*}(v)\right)\right) .
$$

We proceed to show that this is indeed the case.

Recalling (7), we can write $b_{z}^{*}(v)$ as

$$
b_{z}^{*}(v)=A_{z}(v)-B_{z}(v)
$$

where

$$
\begin{aligned}
& A_{z}(v)=\sum_{s=z}^{k}\left(\beta_{s}-\beta_{s+1}\right) \sum_{t=0}^{s-z}(-1)^{t}\left(\begin{array}{c}
s-z \\
t
\end{array}\right) \frac{(n-z) !}{(n-s-1) !(s-z) !} \frac{1}{(n-s+t)} v \quad \text { and } \\
& B_{z}(v)=\sum_{s=z}^{k}\left(\beta_{s}-\beta_{s+1}\right) \sum_{t=0}^{s-z}(-1)^{t}\left(\begin{array}{c}
s-z \\
t
\end{array}\right) \frac{(n-z) !}{(n-s-1) !(s-z) !} \frac{1}{(n-s+t)} Z_{n-s+t}(v) .
\end{aligned}
$$

By applying Lemma 4 for $m=s-z$ and $\ell=n-s$ we see that

$$
A_{z}(v)=\sum_{s=z}^{k}\left(\beta_{s}-\beta_{s+1}\right) v=\beta_{z} v .
$$

For (12) it thus suffices to show that

$$
\left.\frac{d}{d x_{j}} b_{j}^{*}\left(x_{j}\right)\right|_{x_{j}=v}=(n-j) \frac{f(v)}{F(v)}\left(B_{j}(v)-B_{j+1}(v)\right) .
$$

Denoting

$$
C_{z, s, t}(v)=(-1)^{t}\left(\begin{array}{c}
s-z \\
t
\end{array}\right) \frac{(n-z) !}{(n-s-1) !(s-z) !} \frac{1}{(n-s+t)} \frac{f(v)}{F(v)} Z_{n-s+t}(v)
$$

we see that indeed,

$$
\begin{aligned}
(n-j) & \frac{f(v)}{F(v)}\left(B_{j}(v)-B_{j+1}(v)\right) \\
& =(n-j)\left(\sum_{s=j}^{k}\left(\beta_{s}-\beta_{s+1}\right) \sum_{t=0}^{s-j} C_{j, s, t}(v)-\sum_{s=j+1}^{k}\left(\beta_{s}-\beta_{s+1}\right) \sum_{t=0}^{s-j-1} C_{j+1, s, t}(v)\right)
\end{aligned}
$$




$$
\begin{gathered}
=(n-j)\left(\left(\beta_{j}-\beta_{j+1}\right) C_{j, j, 0}(v)+\sum_{s=j+1}^{k}\left(\beta_{s}-\beta_{s+1}\right) \sum_{t=0}^{s-j-1}\left(C_{j, s, t}(v)-C_{j+1, s, t}(v)\right)\right. \\
\left.\quad+\sum_{s=j+1}^{k}\left(\beta_{s}-\beta_{s+1}\right) C_{j, s, s-j}(v)\right) \\
=(n-j)\left(\beta_{j}-\beta_{j+1}\right) C_{j, j, 0}(v)+\sum_{s=j+1}^{k}\left(\beta_{s}-\beta_{s+1}\right) \sum_{t=0}^{s-j-1}(n-s+t) C_{j, s, t}(v) \\
\quad+(n-j) \sum_{s=j+1}^{k}\left(\beta_{s}-\beta_{s+1}\right) C_{j, s, s-j}(v) \\
=\sum_{s=j}^{k}\left(\beta_{s}-\beta_{s+1}\right) \sum_{t=0}^{s-j}(n-s+t) C_{j, s, t}(v)=\left.\frac{d}{d x_{j}} b_{j}^{*}\left(x_{j}\right)\right|_{x_{j}=v},
\end{gathered}
$$

where the second equality holds because

$$
\begin{aligned}
& (n-j)\left(C_{j, s, t}(v)-C_{j+1, s, t}(v)\right) \\
& =(n-j)\left((-1)^{t}\left(\begin{array}{c}
s-j \\
t
\end{array}\right) \frac{(n-j) !}{(n-s-1) !(s-j) !} \frac{1}{(n-s+t)} \frac{f(v)}{F(v)} Z_{n-s+t}(v)-\right. \\
& \left.(-1)^{t}\left(\begin{array}{c}
s-j-1 \\
t
\end{array}\right) \frac{(n-j-1) !}{(n-s-1) !(s-j-1) !} \frac{1}{(n-s+t)} \frac{f(v)}{F(v)} Z_{n-s+t}(v)\right) \\
& =(n-j)\left((-1)^{t}\left(\begin{array}{c}
s-j \\
t
\end{array}\right) \frac{(n-j) !}{(n-s-1) !(s-j) !} \frac{1}{(n-s+t)} \frac{f(v)}{F(v)} Z_{n-s+t}(v)-\right. \\
& \left.(-1)^{t}\left(\begin{array}{c}
s-j \\
t
\end{array}\right) \frac{s-j-t}{s-j} \frac{(n-j-1) !}{(n-s-1) !(s-j-1) !} \frac{1}{(n-s+t)} \frac{f(v)}{F(v)} Z_{n-s+t}(v)\right) \\
& =(-1)^{t}\left(\begin{array}{c}
s-j \\
t
\end{array}\right) \frac{(n-j) !}{(n-s-1) !(s-j) !} \frac{f(v)}{F(v)}\left(\frac{n-j}{n-s+t}-\frac{s-j-t}{n-s+t}\right) Z_{n-s+t}(v) \\
& =(n-s+t) C_{j, s, t}(v)
\end{aligned}
$$

and the last equality follows from (9).

We now turn to the contribution $\sum_{s=j+2}^{k} T_{s}(\boldsymbol{x}, v)$ of positions $s>j+1$.

Lemma 6. Fix a particular agent with value $v$ and a position $j$. Assume that all other agents bid straightforwardly and that the agent bids straightforwardly on positions $j+1, \ldots, k$. Then

$$
\left.\frac{d}{d x_{j}}\left(\sum_{s=j+2}^{k} T_{s}(\boldsymbol{x}, v)\right)\right|_{x_{j}=v}=0 .
$$

For $s>j+1, T_{s}(\boldsymbol{x}, v)=P_{s, n-1}(\boldsymbol{x}, v)\left(\beta_{s} v-b_{s}^{*}\left(x_{s}\right)\right)$ depends on $x_{j}$ only through the allocation probability $P_{s, n-1}(\boldsymbol{x}, v)$. It therefore suffices to show that the derivative in $x_{j}$ of $P_{s, n-1}(\boldsymbol{x}, v)$ vanishes at $x_{j}=v$. We establish this claim with the help of two auxiliary lemmas, which are proved in the appendix and which again exploit the recursive formulation of the allocation probabilities.

Lemma 7. Fix a particular agent with value $v$ and a position $j$. Assume that all other agents bid straightforwardly and that the agent bids straightforwardly on positions $j+1, \ldots, k$. Then, for all $m \geq j+1$,

$$
\left.\frac{d}{d x_{j}}\left(P_{j, m}(\boldsymbol{x})+P_{j+1, m}(\boldsymbol{x})\right)\right|_{x_{j}=v}=0 .
$$


Lemma 8. Fix a particular agent with value $v$ and a position $j$. Assume that all other agents bid straightforwardly and that the agent bids straightforwardly on positions $j+1, \ldots, k$. Then, for all $m$ and $\ell$ with $m \geq \ell \geq j+2$,

$$
\left.\frac{d}{d x_{j}} P_{\ell, m}(\boldsymbol{x})\right|_{x_{j}=v}=0
$$

Proof of Lemma 6. For $s>j+1$

$$
\begin{aligned}
\frac{d}{d x_{j}} T_{s}(\boldsymbol{x}, v) & =\frac{d}{d x_{j}}\left[P_{s, n-1}(\boldsymbol{x}, v)\left(\beta_{s} v-b_{s}^{*}\left(x_{s}\right)\right)\right] \\
& =\frac{d}{d x_{j}}\left[\left(\begin{array}{c}
n-1 \\
n-s
\end{array}\right) F\left(x_{s}\right)^{n-s}\left(1-\sum_{t=1}^{s-1} P_{t, s-1}(\boldsymbol{x})\right)\left(\beta_{s} v-b_{s}^{*}\left(x_{s}\right)\right)\right] \\
& =\frac{d}{d x_{j}}\left[\left(\begin{array}{l}
n-1 \\
n-s
\end{array}\right) F\left(x_{s}\right)^{n-s}\left(1-\sum_{t=1}^{j-1} P_{t, s-1}(\boldsymbol{x})-\sum_{t=j}^{s-1} P_{t, s-1}(\boldsymbol{x})\right)\left(\beta_{s} v-b_{s}^{*}\left(x_{s}\right)\right)\right] \\
& =\left(\begin{array}{l}
n-1 \\
n-s
\end{array}\right) F\left(x_{s}\right)^{n-s}\left(-\frac{d}{d x_{j}} \sum_{t=j}^{s-1} P_{t, s-1}(\boldsymbol{x})\right)\left(\beta_{s} v-b_{s}^{*}\left(x_{s}\right)\right)=0,
\end{aligned}
$$

where the second equality holds by (4), the fourth equality because $P_{t, s-1}(\boldsymbol{x})$ does not depend on $x_{j}$ when $j>t$, and the last equality by Lemma 7 and Lemma 8.

4.4. Proof of Lemma 3. We can again write $u^{*}(\boldsymbol{x}, v)$ as a sum of the contributions $T_{s}(\boldsymbol{x}, v)=$ $P_{s, n-1}(\boldsymbol{x})\left(\beta_{s} v-b_{s}^{*}\left(x_{s}\right)\right)$, and observe that $T_{s}(\boldsymbol{x}, v)$ does not depend on $x_{j}$ when $j>s$, to see that

$$
\frac{d}{d x_{j}} u^{*}(\boldsymbol{x}, v)=\frac{d}{d x_{j}}\left(\sum_{s=1}^{k} T_{s}(\boldsymbol{x}, v)\right)=\frac{d}{d x_{j}}\left(\sum_{s=j}^{k} T_{s}(\boldsymbol{x}, v)\right)=\frac{d}{d x_{j}}\left(T_{j}(\boldsymbol{x}, v)+\sum_{s=j+1}^{k} T_{s}(\boldsymbol{x}, v)\right) .
$$

For the contribution of position $j$,

$$
\begin{aligned}
\frac{d}{d x_{j}} T_{j}(\boldsymbol{x}, v) & =\frac{d}{d x_{j}}\left(P_{j, n-1}(\boldsymbol{x})\left(\beta_{j} v-b_{j}^{*}\left(x_{j}\right)\right)\right) \\
& =\beta_{j} v \frac{d}{d x_{j}} P_{j, n-1}(\boldsymbol{x})-b_{j}^{*}\left(x_{j}\right) \frac{d}{d x_{j}} P_{j, n-1}(\boldsymbol{x})-P_{j, n-1}(\boldsymbol{x}) \frac{d}{d x_{j}} b_{j}^{*}\left(x_{j}\right),
\end{aligned}
$$

and, since $P_{j, n-1}(\boldsymbol{x})$ does not depend on $x_{s}$ for $s>j$,

$$
\frac{d}{d v} \frac{d}{d x_{j}} T_{j}\left(\left(x_{1}, \ldots, x_{j}, v, \ldots, v\right), v\right)=\beta_{j} \frac{d}{d x_{j}} P_{j, n-1}\left(x_{1}, \ldots, x_{j}, v, \ldots, v\right) .
$$

For the contribution of positions $s>j$ we use a variation of the standard technique for one-dimensional settings due to Myerson [35] to write the expected utility as an integral of the expected allocation. Denote $\bar{u}(z, v)=\sum_{s=j+1}^{k} T_{s}\left(\left(x_{1}, \ldots x_{j}, z, \ldots, z\right), v\right)$ and recall that $\bar{u}(z, v)=\bar{x}(z, v) v-\bar{p}(z)$, where $\bar{x}(z, v)=\sum_{s=j+1}^{k} \beta_{s} P_{s, n-1}\left(x_{1}, \ldots, x_{j}, z, \ldots, z\right) v$ and $\bar{p}(z)=$ $\sum_{s=j+1}^{k} \beta_{s} P_{s, n-1}\left(x_{1}, \ldots, x_{j}, z, \ldots, z\right) b_{s}^{*}(z)$. Note further that

$$
\left.\left(\frac{d}{d z} \bar{u}(z, v)\right)\right|_{z=v}=\left.\left(\frac{d}{d z} u^{*}\left(\left(x_{1}, \ldots, x_{j}, z, \ldots, z\right), v\right)\right)\right|_{z=v}=0
$$

where the first equality holds because the expected utility from positions 1 to $j$ is independent of the bid $z$ on positions $j+1$ to $k$, and the second equality by the assumption that for all $\boldsymbol{x}$ and $v$, 
$u^{*}\left(\left(x_{1}, \ldots, x_{j}, v, v, \ldots, v\right), v\right) \geq u^{*}\left(\left(x_{1}, \ldots, x_{k}\right), v\right)$. Since this holds for all values of $v$, it must be the case that

$$
\left(\frac{d}{d z} \bar{x}(z)\right) z=\frac{d}{d z} \bar{p}(z)
$$

and by integrating both sides from 0 to $v$ and setting $\bar{p}(0)=0$ we see that

$$
\bar{p}(v)=\int_{z=0}^{v}\left(\frac{d}{d z} \bar{x}(z)\right) z d z=[\bar{x}(z) z]_{z=0}^{v}-\int_{z=0}^{v} \bar{x}(z) d z=\bar{x}(v) v-\int_{z=0}^{v} \bar{x}(z) d z
$$

and thus

$$
\begin{aligned}
\sum_{s=j+1}^{k} T_{s}\left(\left(x_{1}, \ldots, x_{j}, v, \ldots, v\right), v\right) & =\bar{u}(v, v)=\bar{x}(v) v-\bar{p}(v)=\int_{z=0}^{v} \bar{x}(z) d z \\
& =\sum_{s=j+1}^{k} \beta_{s} \int_{t=0}^{v} P_{s, n-1}\left(x_{1}, \ldots, x_{j}, t, \ldots, t\right) d t
\end{aligned}
$$

By taking derivatives in $x_{j}$ and $v$,

$$
\begin{aligned}
\frac{d}{d v} \frac{d}{d x_{j}} \sum_{s=j+1}^{k} T_{s}\left(\left(x_{1}, \ldots, x_{j}, v, \ldots, v\right), v\right) & =\frac{d}{d v} \frac{d}{d x_{j}}\left(\sum_{s=j+1}^{k} \beta_{s} \int_{t=0}^{v} P_{s, n-1}\left(x_{1}, \ldots, x_{j}, t, \ldots, t\right) d t\right) \\
& =\sum_{s=j+1}^{k} \beta_{s} \frac{d}{d v} \int_{t=0}^{v} \frac{d}{d x_{j}} P_{s, n-1}\left(x_{1}, \ldots, x_{j}, t, \ldots, t\right) d t \\
& =\sum_{s=j+1}^{k} \beta_{s} \frac{d}{d x_{j}} P_{s, n-1}\left(x_{1}, \ldots, x_{j}, v, \ldots, v\right) .
\end{aligned}
$$

Finally

$$
\frac{d}{d v} \frac{d}{d x_{j}} u^{*}\left(\left(x_{1}, \ldots, x_{j}, v, \ldots, v\right), v\right)=\frac{d}{d x_{j}} \sum_{s=j}^{k} \beta_{s} P_{s, n-1}\left(x_{1}, \ldots, x_{j}, v, \ldots, v\right)>0,
$$

where the equality holds by (13), (14), and (15), and the inequality by an ex-post argument: if the agent is currently allocated a position $s \leq j$, then changing its reported valuation $x_{j}$ for position $j$ has no effect and it will still be allocated position $s$; if the agent is currently allocated a position $s>j$ or no position at all, then after increasing $x_{j}$ it will either be allocated the same position as before or position $j$.

5. Conclusion. We have studied a one-dimensional position auction setting and have identified a generalized first-price auction with multi-dimensional bids as the only standard design able to guarantee existence of an efficient equilibrium, and high revenue in every efficient equilibrium, under both complete and incomplete information. That expressiveness beyond that of the valuation space is necessary for robust performance across multiple equilibria and under varying informational assumptions provides a counterpoint to work on position auctions that had highlighted the benefits of simplicity [34, 16]. A conjecture compatible with both lines of work, and also with recent results showing an increased robustness of non-truthful auctions to imprecise modeling by the auctioneer [17], is that simplicity of payment rules rather than simplicity of bids is what drives good performance. This conjecture deserves further investigation.

One may of course wonder whether expressiveness is necessary for robustness in other contexts as well, and whether the techniques we have used to show equilibrium existence can be applied more widely. Natural settings to consider are those with multi-dimensional valuations such as combinatorial auctions, where simplified designs have recently received significant attention $[12,7$, $22,19,4]$, and two-sided markets with strategic buyers and sellers such as assortative matching [28]. 
Appendix A: Proof of Lemma 4 We prove the claim by induction on $\ell$. For $\ell=1$,

$$
\begin{aligned}
\sum_{t=0}^{m}(-1)^{t}\left(\begin{array}{c}
m \\
t
\end{array}\right) \frac{(m+\ell) !}{(\ell-1) ! m !(t+\ell)} & =\sum_{t=0}^{m}(-1)^{t}\left(\begin{array}{c}
m \\
t
\end{array}\right) \frac{(m+1) !}{0 ! m !(t+1)} \\
& =\sum_{t=0}^{m}(-1)^{t}\left(\begin{array}{c}
m+1 \\
t+1
\end{array}\right) \\
& =\sum_{t=1}^{m+1}(-1)^{t-1}\left(\begin{array}{c}
m+1 \\
t
\end{array}\right) \\
& =1-\sum_{t=0}^{m+1}(-1)^{t}\left(\begin{array}{c}
m+1 \\
t
\end{array}\right) \\
& =1-(1-1)^{m+1}=1,
\end{aligned}
$$

where the penultimate equality holds by the binomial theorem.

Now let $\ell \geq 2$ and assume that the claim holds for $\ell-1$, i.e., that

$$
\sum_{t=0}^{m}(-1)^{t}\left(\begin{array}{c}
m \\
t
\end{array}\right) \frac{(m+\ell-1) !}{(\ell-2) ! m !(t+\ell-1)}=1 \text {. }
$$

Then

$$
\begin{aligned}
\sum_{t=0}^{m}(-1)^{t} & \left(\begin{array}{c}
m \\
t
\end{array}\right) \frac{(m+\ell) !}{(\ell-1) ! m !(t+\ell)} \\
& =\sum_{t=0}^{m}(-1)^{t} \frac{m !(m+\ell) !}{t !(m-t) !(\ell-1) ! m !(t+\ell)}=\sum_{t=0}^{m}(-1)^{t}\left(\begin{array}{c}
m+\ell \\
t+\ell
\end{array}\right) \frac{\prod_{s=1}^{\ell-1}(t+s)}{(\ell-1) !} \\
& =\sum_{t=0}^{m}(-1)^{t}\left(\begin{array}{c}
m+\ell-1 \\
t+\ell-1
\end{array}\right) \frac{\prod_{s=1}^{\ell-1}(t+s)}{(\ell-1) !}+\sum_{t=0}^{m}(-1)^{t}\left(\begin{array}{c}
m+\ell-1 \\
t+\ell
\end{array}\right) \frac{\prod_{s=1}^{\ell-1}(t+s)}{(\ell-1) !} \\
& =\sum_{t=0}^{m}(-1)^{t}\left(\begin{array}{c}
m+\ell-1 \\
t+\ell-1
\end{array}\right) \frac{\prod_{s=1}^{\ell-2}(t+s)}{(\ell-2) !}\left(1+\frac{t}{\ell-1}\right)+\sum_{t=1}^{m+1}(-1)^{t-1}\left(\begin{array}{c}
m+\ell-1 \\
t+\ell-1
\end{array}\right) \frac{\prod_{s=0}^{\ell-2}(t+s)}{(\ell-1) !} \\
& =\sum_{t=0}^{m}(-1)^{t}\left(\begin{array}{c}
m+\ell-1 \\
t+\ell-1
\end{array}\right) \frac{\prod_{s=1}^{\ell-2}(t+s)}{(\ell-2) !}+\sum_{t=0}^{m}(-1)^{t}\left(\begin{array}{c}
m+\ell-1 \\
t+\ell-1
\end{array}\right) \frac{\prod_{s=0}^{\ell-2}(t+s)}{(\ell-1) !} \\
& =\sum_{t=0}^{m}(-1)^{t}\left(\begin{array}{c}
m+\ell-1 \\
t+\ell-1
\end{array}\right) \frac{\prod_{s=1}^{\ell-2}(t+s)}{(\ell-2) !}=\sum_{t=0}^{m}(-1)^{t} \frac{(m+\ell-1) !}{(t+\ell-1) !(m-t) !(\ell-2) !} \\
& =\sum_{t=0}^{m}(-1)^{t}\left(\begin{array}{c}
m \\
t
\end{array}\right) \frac{(m+-1)^{t-1}\left(\begin{array}{c}
m+\ell-1 \\
t+\ell-1
\end{array}\right) \frac{\prod_{s=0}^{\ell-2}(t+s)}{(\ell-1) !}}{(\ell-2) !(t+\ell) !}
\end{aligned}
$$

where the third equality holds by Pascal's identity and the last equality by (16).

Appendix B: Proof of Lemma 7. We consider the derivatives of $P_{j, m}(\boldsymbol{x})$ and $P_{j+1, m}(\boldsymbol{x})$ in turn. For $P_{j, m}(\boldsymbol{x})$,

$$
\frac{d}{d x_{j}} P_{j, m}(\boldsymbol{x})=\frac{d}{d x_{j}}\left[\left(\begin{array}{c}
m \\
m-j+1
\end{array}\right) F\left(x_{j}\right)^{m-j+1}\left(1-\sum_{t=1}^{j-1} P_{t, j-1}(\boldsymbol{x})\right)\right]
$$




$$
=\left(\begin{array}{c}
m \\
m-j+1
\end{array}\right)(m-j+1) F\left(x_{j}\right)^{m-j} f\left(x_{j}\right)\left(1-\sum_{t=1}^{j-1} P_{t, j-1}(\boldsymbol{x})\right),
$$

where the first equality holds by (4) and the second equality because $P_{t, j-1}(\boldsymbol{x})$ does not depend on $x_{j}$ when $t<j$. For $P_{j+1, m}(\boldsymbol{x})$,

$$
\begin{aligned}
\frac{d}{d x_{j}} P_{j+1, m}(\boldsymbol{x}) & =\frac{d}{d x_{j}}\left[\left(\begin{array}{c}
m \\
m-j
\end{array}\right) F(v)^{m-j}\left(1-\sum_{t=1}^{j} P_{t, j}(\boldsymbol{x})\right)\right] \\
& =\frac{d}{d x_{j}}\left[\left(\begin{array}{c}
m \\
m-j
\end{array}\right) F(v)^{m-j}\left(1-\sum_{t=1}^{j-1} P_{t, j}(\boldsymbol{x})-\left(\begin{array}{c}
j \\
1
\end{array}\right) F\left(x_{j}\right)\left(1-\sum_{t=1}^{j-1} P_{t, j-1}(\boldsymbol{x})\right)\right)\right] \\
& =\left(\begin{array}{c}
m \\
m-j
\end{array}\right) F(v)^{m-j}\left(-\left(\begin{array}{l}
j \\
1
\end{array}\right) f\left(x_{j}\right)\left(1-\sum_{t=1}^{j-1} P_{t, j-1}(\boldsymbol{x})\right)\right),
\end{aligned}
$$

where the first equality holds by (4), the second equality is obtained by pulling $P_{j, j}(\boldsymbol{x})$ out of the sum and applying (4) again, and the third equality holds because $P_{t, j}(\boldsymbol{x})$ and $P_{t, j-1}(\boldsymbol{x})$ do not depend on $x_{j}$ when $t<j$. Thus

$$
\begin{aligned}
\left.\frac{d}{d x_{j}}\left(P_{j, m}(\boldsymbol{x})+P_{j+1, m}(\boldsymbol{x})\right)\right|_{x_{j}=v} & =\left(\begin{array}{c}
m \\
m-j+1
\end{array}\right)(m-j+1) F(v)^{m-j} f(v) \\
& =0, \quad-\left(\begin{array}{c}
m \\
m-j
\end{array}\right) F(v)^{m-j}\left(\begin{array}{l}
j \\
1
\end{array}\right) f(v)
\end{aligned}
$$

where the second equality holds because $\left(\begin{array}{c}m \\ m-j\end{array}\right)\left(\begin{array}{l}j \\ 1\end{array}\right)=\left(\begin{array}{c}m \\ m-j+1\end{array}\right)(m-j+1)$.

Appendix C: Proof of Lemma 8. We prove the claim by induction on $m$. When $m=j+2$, the only possible value for $\ell$ is $j+2$ and

$$
\begin{aligned}
\frac{d}{d x_{j}} P_{j+2, j+2}(\boldsymbol{x}) & =\frac{d}{d x_{j}}\left[\left(\begin{array}{c}
j+2 \\
1
\end{array}\right) F(v)\left(1-\sum_{t=1}^{j+1} P_{t, j+1}(\boldsymbol{x})\right)\right] \\
& =\frac{d}{d x_{j}}\left[\left(\begin{array}{c}
j+2 \\
1
\end{array}\right) F(v)\left(1-\sum_{t=1}^{j-1} P_{t, j+1}(\boldsymbol{x})-\left(P_{j, j+1}(x)+P_{j+1, j+1}(\boldsymbol{x})\right)\right)\right] \\
& =-\left(\begin{array}{c}
j+2 \\
1
\end{array}\right) F(v) \frac{d}{d x_{j}}\left(P_{j, j+1}(\boldsymbol{x})+P_{j+1, j+1}(\boldsymbol{x})\right),
\end{aligned}
$$

where the first equality holds by (4) and the third equality because $P_{t, j+1}(\boldsymbol{x})$ does not depend on $x_{j}$ when $t<j$. By Lemma $7,\left.\frac{d}{d x_{j}}\left(P_{j, j+1}(\boldsymbol{x})+P_{j+1, j+1}(\boldsymbol{x})\right)\right|_{x_{j}=v}=0$, and thus $\left.\frac{d}{d x_{j}} P_{j+2, j+2}(\boldsymbol{x})\right|_{x_{j}=v}=0$.

Now consider $m>j+2$ and assume that for all $m^{\prime}$ and $\ell$ such that $m>m^{\prime} \geq \ell \geq j+2$, $\left.\frac{d}{d x_{j}} P_{\ell, m^{\prime}}(\boldsymbol{x})\right|_{x_{j}=v}=0$. Then, for $m$ and $\ell$ with $m \geq \ell \geq j+2$,

$$
\begin{aligned}
\frac{d}{d x_{j}} P_{\ell, m}(\boldsymbol{x}) & =\frac{d}{d x_{j}}\left[\left(\begin{array}{c}
m \\
m-\ell+1
\end{array}\right) F(v)^{m-\ell+1}\left(1-\sum_{t=1}^{\ell-1} P_{t, \ell-1}(\boldsymbol{x})\right)\right] \\
& =\frac{d}{d x_{j}}\left[\left(\begin{array}{c}
m \\
m-\ell+1
\end{array}\right) F(v)^{m-\ell+1}\left(1-\sum_{t=1}^{j-1} P_{t, \ell-1}(\boldsymbol{x})-\sum_{t=j}^{j+1} P_{t, \ell-1}(\boldsymbol{x})-\sum_{t=j+2}^{\ell-1} P_{t, \ell-1}(\boldsymbol{x})\right)\right] \\
& =-\left(\begin{array}{c}
m \\
m-\ell+1
\end{array}\right) F(v)^{m-\ell+1}\left(\frac{d}{d x_{j}} \sum_{t=j}^{j+1} P_{t, \ell-1}(\boldsymbol{x})+\frac{d}{d x_{j}} \sum_{t=j+2}^{\ell-1} P_{t, \ell-1}(\boldsymbol{x})\right),
\end{aligned}
$$


where the third equality holds because $P_{t, \ell-1}(\boldsymbol{x})$ does not depend on $x_{j}$ when $t<j$. Since $\ell-1 \geq$ $j+1$, by Lemma 7 ,

$$
\left.\left(\frac{d}{d x_{j}} \sum_{t=j}^{j+1} P_{t, \ell-1}(\boldsymbol{x})\right)\right|_{x_{j}=v}=0 .
$$

Moreover $\ell-1 \leq m-1<m$, so by the induction hypothesis,

$$
\left.\left(\frac{d}{d x_{j}} \sum_{t=j+2}^{\ell-1} P_{t, \ell-1}(\boldsymbol{x})\right)\right|_{x_{j}=v}=0 .
$$

Thus $\left.\frac{d}{d x_{j}} P_{\ell, m}(\boldsymbol{x})\right|_{x_{j}=v}=0$, as claimed.

Acknowledgments. We thank Jason Hartline, Robert Kleinberg, Benny Moldovanu, Éva Tardos, and the anonymous referees for their valuable feedback. The first author was supported in part by an SNF Postdoctoral Fellowship, the second author by the Einstein Foundation Berlin.

\section{References}

[1] Abrams, Z., A. Ghosh, E. Vee. 2009. Cost of conciseness in sponsored search auctions. Proceedings of the 5th Workshop on Internet and Network Economics. 326-334.

[2] Aggarwal, G., S. Muthukrishnan, D. Pál, M. Pál. 2009. General auction mechanism for search advertising. Proceedings of the 18th International Conference on World Wide Web. 241-250.

[3] Ausubel, L., P. Milgrom. 2002. Ascending auctions with package bidding. The B.E. Journal of Theoretical Economics 1(1) Article 1.

[4] Babaioff, M., B. Lucier, R. Paes Leme, N. Nisan. 2014. On the efficiency of the Walrasian mechanism. Proceedings of the 15th ACM Conference on Economics and Computation. 783-800.

[5] Benisch, M., N. Sadeh, T. Sandholm. 2008. A theory of expressiveness in mechanisms. Proceedings of the 23rd AAAI Conference on Artificial Intelligence. 17-23.

[6] Bernheim, B. D., M. D. Whinston. 1986. Menu auctions, resource allocation and economic influence. Quarterly Journal of Economics 101(1) 1-31.

[7] Bhawalkar, K., T. Roughgarden. 2011. Welfare guarantees for combinatorial auctions with item bidding. Proceedings of the 22nd Annual ACM-SIAM Symposium on Discrete Algorithms. 700-709.

[8] Blumrosen, L., J. Hartline, S. Nong. 2008. Position auctions and non-uniform conversion rates. Proceedings of the 8th Ad Auctions Workshop. 1-11.

[9] Caragiannis, I., C. Kaklamanis, P. Kanellopoulos, M. Kyropoulou. 2012. Revenue guarantees in sponsored search auctions. Proceedings of the 20th European Symposium on Algorithms. 253-264.

[10] Caragiannis, I., C. Kaklamanis, P. Kanellopoulos, M. Kyropoulou, B. Lucier, R. Paes Leme, É. Tardos. 2015. Bounding the inefficiency of outcomes in generalized second price auctions. Journal of Economic Theory 156 343-388.

[11] Chawla, S., J. Hartline. 2013. Auctions with unique equilibria. Proceedings of the 14th ACM Conference on Electronic Commerce. 181-196.

[12] Christodoulou, G., A. Kovács, M. Schapira. 2008. Bayesian combinatorial auctions. Proceedings of the 35th International Colloquium on Automata, Languages and Programming. 820-832.

[13] Constantin, F., M. Rao, C.-C. Huang, D. C. Parkes. 2011. On expressing value externalities in position auctions. Proceedings of the 25th AAAI Conference on Artificial Intelligence. 644-649.

[14] Day, R., P. Milgrom. 2008. Core-selecting package auctions. International Journal of Game Theory 36(3) 393-407.

[15] Dobzinski, S., R. Lavi, N. Nisan. 2012. Multi-unit auctions with budget limits. Games and Economic Behavior 74(2) 486-503. 
[16] Dütting, P., F. Fischer, D. C. Parkes. 2011. Simplicity-expressiveness tradeoffs in mechanism design. Proceedings of the 12th ACM Conference on Electronic Commerce. 341-350.

[17] Dütting, P., F. Fischer, D. C. Parkes. 2016. Truthful outcomes from non-truthful position auctions. Proceedings of the 17th ACM Conference on Economics and Computation. ACM Press, 813.

[18] Dütting, P., M. Henzinger, M. Starnberger. 2012. Auctions for heterogeneous items and budget limits. Proceedings of the 8th Workshop on Internet and Network Economics. 44-57.

[19] Dütting, P., M. Henzinger, M. Starnberger. 2013. Valuation compressions in VCG-based combinatorial auctions. Proceedings of the 9th Conference on Web and Internet Economics. 146-159.

[20] Dütting, P., M. Henzinger, I. Weber. 2015. An expressive mechanism for auctions on the web. ACM Transactions on Economics and Computation 4(1) Article 1.

[21] Edelman, B., M. Ostrovsky, M. Schwartz. 2007. Internet advertising and the generalized second price auction: Selling billions of dollars worth of keywords. American Economic Review 97(1) 242-259.

[22] Feldman, M., H. Fu, N. Gravin, B. Lucier. 2013. Simultaneous auctions are (almost) efficient. Proceedings of the 45th Annual ACM Symposium on Theory of Computing. 201-210.

[23] Ghosh, A., A. Sayedi. 2010. Expressive auctions for externalities in online advertising. Proceedings of the 19th International Conference on World Wide Web. 371-380.

[24] Goel, G., V. S. Mirrokni, R. Paes Leme. 2015. Polyhedral clinching auctions and the adwords polytope. Journal of the ACM 62(3) Article 18.

[25] Gomes, R., K. S. Sweeney. 2014. Bayes-Nash equilibria of the generalized second-price auction. Games and Economic Behavior 86 421-437.

[26] Gul, F., E. Stacchetti. 1999. Walrasian equilibrium with gross substitutes. Journal of Economic Theory 87(1) 95-124.

[27] Hassidim, A., H. Kaplan, Y. Mansour, N. Nisan. 2011. Non-price equilibria in markets of discrete goods. Proceedings of the 12th ACM Conference on Electronic Commerce. 295-296.

[28] Hoppe, H. C., B. Moldovanu, A. Sela. 2009. The theory of assortative matching based on costly signals. Review of Economic Studies 76(1) 253-281.

[29] Hoy, D., K. Jain, C. A. Wilkens. 2013. A dynamic axiomatic approach to first-price auctions. Proceedings of the 14th ACM Conference on Electronic Commerce. 583-584.

[30] Krishna, V., M. Perry. 2000. Efficient mechanism design. Manuscript.

[31] Leonard, H. B. 1983. Elicitation of honest preferences for the assignment of individuals to positions. Journal of Political Economy 91(3) 461-479.

[32] Lucier, B., R. Paes Leme, É. Tardos. 2012. On revenue in the generalized second price auction. Proceedings of the 21st International Conference on World Wide Web. 361-370.

[33] Milgrom, P. 2004. Putting Auction Theory to Work. Cambridge University Press.

[34] Milgrom, P. 2010. Simplified mechanisms with an application to sponsored-search auctions. Games and Economic Behavior 70(1) 62-70.

[35] Myerson, R. 1981. Optimal auction design. Mathematics of Operations Research 6 58-73.

[36] Syrgkanis, V., É. Tardos. 2013. Composable and efficient mechanisms. Proceedings of the 45th Annual ACM Symposium on Theory of Computing. 211-220.

[37] Varian, H. 2007. Position auctions. International Journal of Industrial Organization 25 1163-1178. 